\title{
Évaluation de la sensibilité du laminage aux modèles de comportement
}

\author{
Mohamed ZaAF ${ }^{1,2, a}$, Mohamed Labaiz ${ }^{1}$ et François SidorofF ${ }^{2}$ \\ 1 Laboratoire de Métallurgie et Génie des Matériaux, Université Badji-Mokhtar, Annaba, Algérie \\ ${ }^{2}$ Laboratoire de Tribologie et Dynamique des Systèmes, UMR CNRS 5513, École Centrale de Lyon, 36 avenue Gug de Collonge, \\ 69134 Écully Cedex, France
}

Reçu le 27 juillet 2004, accepté le 17 mai 2005

\begin{abstract}
Résumé - Le travail présenté ici porte sur l'application au laminage des modèles de comportement récemment développés en plasticité grandes déformations en vue de la simulation numérique des procédés de mise en forme et notamment de l'emboutissage : modèle rigide plastique avec écrouissage isotrope, critère de Hill quadratique ou non-quadratique, plasticité associée ou non, viscoplasticité. Après avoir brièvement présenté ces modèles nous les intégrons dans le modèle classique de laminage (méthode des tranches, équation de Karman). La solution est obtenue en déterminant le point neutre par une méthode de tir. Ces modélisations sont ensuite illustrées par une analyse paramétrique de l'influence des divers paramètres constitutifs sur la réponse et une comparaison sur un matériau donné de différents modèles en exploitant les modèles identifiés par A. Khalfallah, A. Znaidi et A. Darsouni finalise ce travail.
\end{abstract}

Mots clés : Lois de comportement / laminage / mise en forme des métaux / modélisation

\begin{abstract}
Sensibility evaluation of rolling with behaviour models. The present work is devoted to the application of anisotropic plastic models to the analysis of the rolling process. Many models have been developed in recent years for large strain plasticity in connection with metal forming and in particular deepdrawing: anisotropic plasticity with isotropic hardening, Hill's quadratic or non-quadratic yield function, associated or non-associated flow rules. After a brief presentation of these models, their application to the standard rolling model (one dimensional method, Karman equation) is developed; the solution is obtained by determination of the neutral point through a shooting method: These models are then exemplified by a) a parametric analysis of the influence of the constitutive parameters on the response. b) a comparison for a given material of different models as identified by A. Khalfallah, A. Znaidi and A. Darsouni.
\end{abstract}

Key words: Behaviour lows / rolling / metal's forming / modeling

\section{Introduction}

Les progrès combinés de l'outil informatique et de la mécanique des matériaux ont conduit à des progrès spectaculaires dans la modélisation du comportement plastique en grandes déformations et dans son application à la mise en forme des métaux, conduisant ainsi à une meilleure maîtrise de ces procédés. C'est ainsi que les problèmes difficiles liés notamment à la prise en compte de l'anisotropie sont aujourd'hui relativement bien compris.

a Auteur correspondant : zaaf_mohamed@yahoo.fr
L'essentiel de ces travaux a été jusqu'à présent mené dans le cadre de l'emboutissage. L'objectif du travail que nous présentons ici est de mettre en œuvre les modèles ainsi développés dans le cadre de la modélisation classique du laminage et de permettre ainsi d'apprécier les poids respectifs de divers raffinements proposés pour la loi de comportement (anisotropie, critère non-quadratique, plasticité non-associée).

Comme tout procédé de mise en forme, le laminage implique des déformations très importantes (jusqu'à $90 \%$ ). Sa modélisation exige donc a priori l'utilisation d'un formalisme grandes déformations, formalisme aujourd'hui 
bien maîtrisé. Un élément important est l'utilisation du formalisme en référentiel tournant qui permet d'assurer l'objectivité de la loi de comportement [1]. Cependant, il est avéré que si la rotation des axes principaux reste faible et à condition d'utiliser le tenseur des déformations logarithmiques $h=1 / 2 \log B$ (tenseur de Hencky), un formalisme petites déformations reste valable sans erreurs conséquentes [2]. Cette approximation est en première approximation valable pour le laminage des produits plats. Nous nous plaçons désormais dans ce cadre. De plus, et comme il est classique en mise en forme nous nous limiterons à un écrouissage isotrope et à un modèle rigide plastique.

Après avoir brièvement présenté à la section 2 les divers modèles de comportement qui seront utilisés dans la suite, nous adapterons à la section 3 le modèle classique de laminage (modèle monodimensionnel, méthode des tranches [3]) à ces modèles de comportement, nous intégrerons également le cas viscoplastique. Nous présenterons ensuite les résultats ainsi obtenus dans le cadre d'une analyse paramétrique de l'influence des divers paramètres et hypothèses (Sect. 4) puis plus concrètement aux modèles identifiés expérimentalement par A. Znaidi, A. Khalfallah et A. Darsouni.

\section{Modèles de comportement}

\subsection{Formalisme général}

Comme défini plus haut, nous considérons un modèle rigide plastique avec écrouissage isotrope. Le comportement est donc défini par les lois suivantes.

$$
\begin{cases}\dot{\varepsilon}_{i j}=0 & \text { si } f\left(\sigma_{i j}\right)-Y(p)<0 \\ \dot{\varepsilon}_{i j}=\lambda \frac{\partial g}{\partial \sigma_{i j}} & \text { si } f\left(\sigma_{i j}, Y\right)=f\left(\sigma_{i j}\right)-Y=0\end{cases}
$$

Elles font intervenir la fonction seuil $f\left(\sigma_{i j}\right)$, le potentiel plastique $g\left(\sigma_{i j}\right)$ qui cö̈ncidera avec la fonction seuil dans le cas de la plasticité associée et la loi d'écrouissage $Y(p)$ où $p$ est la variable d'écrouissage isotrope $(\lambda=$ p) $[2]$.

Le modèle classique utilisé pour la description du laminage correspond dans ce cadre à la plasticité associée avec critère de Von Mises.

$$
\begin{gathered}
f=g=\bar{\sigma}=\frac{\sqrt{3}}{2}|S| \\
|S|=\sqrt{S_{i j} S_{i j}} \\
\left\{\begin{array}{l}
\dot{\varepsilon}_{i j}=0 \quad \text { si } \quad \bar{\sigma}-Y(p)<0 \\
\dot{\varepsilon}_{i j}=\frac{3}{2} \lambda \frac{S_{i j}}{\bar{\sigma}} \quad \text { si } \bar{\sigma}-Y(p)=0 \\
\lambda=\dot{p}=\sqrt{\frac{2}{3}} \sqrt{\dot{\varepsilon}_{i j} \dot{\varepsilon}_{i j}}
\end{array}\right.
\end{gathered}
$$

Ce qui permet d'interpréter la variable d'écrouissage isotrope comme étant la déformation plastique cumulée équivalente de Von Mises. Les travaux récents en plasticité grandes déformations ont montré la nécessité d'étendre ce modèle au cas de la plasticité anisotrope, associée ou non et parfois avec un critère de Hill nonquadratique. On trouvera dans [4] et [5] une présentation détaillée de ces modèles et de leurs utilisations, notamment en emboutissage.

\subsection{Cadre orthotrope}

Dans le cas anisotrope on utilise habituellement un critère quadratique par rapport aux contraintes, ce qui revient à utiliser une contrainte équivalente $\hat{\sigma}=$ $\sqrt{H_{i j k l} \sigma_{k l} \sigma_{i j}}$ où le tenseur du $4^{\mathrm{e}}$ ordre $H$ caractérise l'anisotropie. Dans le cas orthotrope et en supposant le critère insensible à la pression hydrostatique on obtient le critère classique de Hill qui lorsque le tenseur des contraintes est diagonal dans le repère d'orthotropie (ce qui sera en première approximation le cas du laminage) s'écrira comme suit :

$$
\hat{\sigma}=\sqrt{F\left(\sigma_{2}-\sigma_{3}\right)+G\left(\sigma_{3}-\sigma_{1}\right)^{2}+H\left(\sigma_{1}-\sigma_{2}\right)^{2}}
$$

Elle fait intervenir trois coefficients $F, G$ et $H$ qui en fait n'interviennent que par leur rapport $\frac{H}{G}$ et $\frac{H}{F}$.

En normant les constantes de manière à ce que la contrainte équivalente coïncide avec la contrainte appliquée dans un essai en traction simple suivant la direction de laminage et en posant $r_{1}=\frac{H}{G}$ et $r_{2}=\frac{H}{F}$ et en choisissant $G+H=1$ on peut écrire :

$\hat{\sigma}=$

$$
\frac{1}{\sqrt{1+r_{1}}} \sqrt{\frac{r_{1}}{r_{2}}\left(\sigma_{2}-\sigma_{3}\right)^{2}+\left(\sigma_{3}-\sigma_{1}\right)^{2}+r_{1}\left(\sigma_{1}-\sigma_{2}\right)^{2}}
$$

En plasticité associée $f=g$ et on peut vérifier la variable d'écrouissage isotrope $p$ à la déformation équivalente cumulée $\hat{\varepsilon}$.

$$
\hat{\varepsilon}=\sqrt{h_{i j k l} \varepsilon_{k l} \varepsilon_{i j}}
$$

où le tenseur du quatrième ordre $h$ est l'inverse de $H$ (ou plus exactement de sa restriction à l'ensemble des déviateurs).

Compte tenu du fait que l'influence de l'anisotropie est en général beaucoup plus sensible sur la loi d'évolution que sur la fonction seuil, il est souvent utile d'utiliser un modèle de plasticité non-associée. On prend alors le potentiel plastique $g$ sous une forme analogue à $f$ mais en utilisant les coefficients de Lankford mesurés expérimentalement $\bar{r}_{1}$ et $\bar{r}_{2}$.

Dans ce cas la variable d'écrouissage $p$ toujours définie par $\dot{p}=\lambda$ devient [2] :

$\dot{p}=$

$$
\left\{\frac{\bar{F}\left(\bar{H} \dot{\varepsilon}_{3}-\bar{G} \dot{\varepsilon}_{2}\right)^{2}+\bar{G}\left(\bar{F} \dot{\varepsilon}_{1}-\bar{H} \dot{\varepsilon}_{3}\right)^{2}+\bar{H}\left(\bar{G} \dot{\varepsilon}_{2}-\bar{F} \dot{\varepsilon}_{1}\right)^{2}}{(\bar{F} \bar{G}+\bar{G} \bar{H}+\bar{H} \bar{F})^{2}}\right\}^{1 / 2}
$$




$$
\dot{p}=R \sqrt{\left(\bar{r}_{2} \bar{r}_{1}^{2} \dot{\varepsilon}_{3}-\bar{r}_{2} \bar{r}_{1} \dot{\varepsilon}_{2}\right)^{2}+\left(\bar{r}_{2} \bar{r}_{1} \dot{\varepsilon}_{1}-\bar{r}_{2}^{2} \bar{r}_{1} \dot{\varepsilon}_{3}\right)^{2}+\left(\bar{r}_{2}^{2} \bar{r}_{1} \dot{\varepsilon}_{2}-\bar{r}_{2} \bar{r}_{1}^{2} \dot{\varepsilon}_{1}\right)^{2}}
$$

De même que pour l'équation (6), on obtient (8)

$$
\text { voir l'équation (8) ci-dessus }
$$

où

$$
R=\frac{\sqrt{\bar{r}_{2}+\bar{r}_{2} \bar{r}_{1}}}{\bar{r}_{2} \bar{r}_{1}+\bar{r}_{2}^{2} \bar{r}_{1}+\bar{r}_{2} \bar{r}_{1}^{2}}
$$

\subsection{Cadre isotrope transverse}

Très souvent, du moins en emboutissage, l'anisotropie se manifeste par un coefficient de Lankford différent de 1 mais ne dépendant que faiblement de la direction dans laquelle est mené l'essai de traction. Ceci conduit souvent à utiliser et identifier un modèle isotrope transverse et ne faisant donc intervenir qu'un seul coefficient de Lankford (deux en plasticité non-associée).

$$
f\left(\sigma_{i j}\right)=\frac{1}{\sqrt{1+r}} \sqrt{\left(\sigma_{2}-\sigma_{3}\right)^{2}+\left(\sigma_{3}-\sigma_{1}\right)^{2}+r\left(\sigma_{1}-\sigma_{2}\right)^{2}}
$$

$$
g\left(\sigma_{i j}\right)=\frac{1}{\sqrt{1+\bar{r}}} \sqrt{\left(\sigma_{2}-\sigma_{3}\right)^{2}+\left(\sigma_{3}-\sigma_{1}\right)^{2}+\bar{r}\left(\sigma_{1}-\sigma_{2}\right)^{2}}
$$

\subsection{Critères non-quadratiques}

Des travaux récents en plasticité grandes déformations ont montré qu'il est possible d'identifier le comportement plastique avec plus de précision en utilisant des critères non-quadratiques $[4,5]$.

Aussi, Hershey (1954) et Hosford (1972) ont introduit un critère isotrope non-quadratique exprimé dans les axes de l'orthotropie particulièrement convenable pour tenir compte des structures cristallographiques, cubiques centrées et cubiques à faces centrées des matériaux isotropes. Ce critère se présente formellement comme une extension non-quadratique du critère de Von Mises sous la forme

$$
\widehat{\sigma}-\left\{\left|\sigma_{1}-\sigma_{2}\right|^{m}+\left|\sigma_{2}-\sigma_{3}\right|^{m}+\left|\sigma_{3}-\sigma_{1}\right|^{m}\right\}^{\frac{1}{m}}=0
$$

Hill (1979) a proposé un critère orthotrope nonquadratique applicable uniquement dans le cas où les axes principaux des contraintes coïncident avec les directions d'orthotropie. Ceci est le cas du laminage. L'expression de ce critère s'exprime de la façon suivante :

$\widehat{\sigma}-\left\{F\left|\sigma_{1}-\sigma_{2}\right|^{m}+G\left|\sigma_{2}-\sigma_{3}\right|^{m}+H\left|\sigma_{3}-\sigma_{1}\right|^{m}\right\}^{\frac{1}{m}}=0$

La contrainte équivalente s'écrit donc

$\widehat{\sigma}=$

$$
\frac{1}{\sqrt[m]{1+r}} \sqrt[m]{\frac{r_{1}}{r_{2}}\left(\sigma_{2}-\sigma_{3}\right)^{m}+\left(\sigma_{3}-\sigma_{1}\right)^{m}+r_{1}\left(\sigma_{1}-\sigma_{2}\right)^{m}}
$$

\subsection{Lois d'écrouissage}

Les lois d'écrouissage les plus classiquement utilisées sont :

Loi puissance ou loi d'Hollomon

$$
Y(p)=K p^{n}
$$

Loi de Ludwick

$$
Y(p)=\sigma_{0}+K p^{n}
$$

Loi de Swift

$$
Y(p)=K\left(\varepsilon_{0}+p\right)^{n}
$$

L'identification des coefficients $K, n, \varepsilon_{0}$ et $\sigma_{0}$ se fait suite à des essais expérimentaux (en général, des essais de traction simple suffisent).

\subsection{Cadre viscoplastique}

Dans le cadre viscoplastique la fonction seuil sera remplacée par un potentiel de dissipation et on écrira :

$$
\sigma_{i j}=\frac{\partial \Omega}{\partial \dot{\varepsilon}_{i j}}
$$

Le potentiel $\Omega$ est donné pour le modèle de NortonHoff par

$$
\begin{aligned}
& \Omega=\frac{A}{m+1} \dot{\hat{\varepsilon}}^{m+1} \\
& \hat{\sigma}=A \dot{\hat{\varepsilon}}^{m}
\end{aligned}
$$

forme générale valable dans le cas isotrope comme dans le cas anisotrope. Toutefois en laminage à chaud l'anisotropie est en général négligeable et nous nous limiterons alors au cas isotrope et on utilisera donc les contraintes et les déformations équivalentes de Von Mises. La prise en compte de l'écrouissage se fera alors simplement en généralisant la dernière relation sous la forme.

$$
\begin{aligned}
\hat{\sigma} & =A \dot{\bar{\varepsilon}}^{m} p^{n} \\
\dot{p} & =\dot{\bar{\varepsilon}}
\end{aligned}
$$

\section{Application au laminage}

\section{1 Équation de Karman}

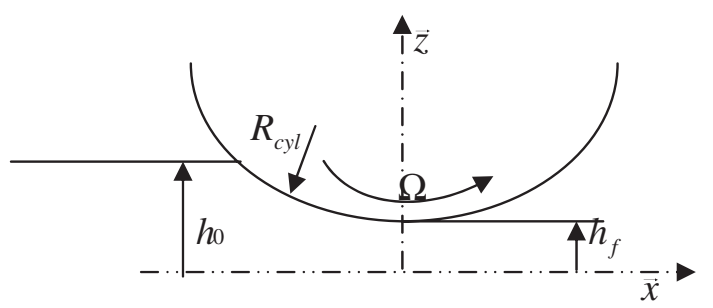


Notre modèle s'appuie classiquement sur la méthode des tranches [3] et suppose que la déformation dominante est un cisaillement pur (extension dans la direction $x$, écrasement dans la direction $z$ ). L'équilibre horizontal et les hypothèses $\frac{\mathrm{d} h}{\mathrm{~d} x} \ll 1, \dot{\varepsilon}_{X Z} \ll \dot{\varepsilon}_{X X}, \sigma_{i j}=\sigma_{i j}(x)$ nous permettent alors d'écrire

$$
h \frac{\mathrm{d} \sigma_{1}}{\mathrm{~d} x}=\left(\sigma_{1}-\sigma_{3}\right) \frac{\mathrm{d} h}{\mathrm{~d} x}-\tau
$$

où

$h$ est la demi-hauteur de la tranche

$\sigma_{1}$ est la contrainte longitudinale (direction $x$ )

$\sigma_{3}$ est la contrainte normale (direction $z$ ) assimilée à la pression de contact

$\tau$ la contrainte de frottement.

Cette équation doit être complétée par deux équations supplémentaires traduisant d'une part la loi de comportement qui donnera une relation entre $\left(\sigma_{1}-\sigma_{3}\right)$ et $\varepsilon_{3}$ (ou $\dot{\varepsilon}_{3}$ dans le cas viscoplastique), où d'après l'incompressibilité plastique $\varepsilon_{3}=-\varepsilon_{1}=\log \frac{h}{h_{0}}$ et d'autre part une loi de frottement.

La relation traduisant la loi de comportement sera explicitée à la section 3.2 pour les différents modèles discutés plus haut.

La loi de frottement sera postulée sous la forme d'une loi de Coulomb ou de Tresca si elle est indépendante visà-vis des vitesses $[6,7]$.

Coulomb donne

$$
\tau=-\min \left\{\mu \sigma_{n}, \frac{\sigma_{0}}{\sqrt{3}}\right\} \frac{\vec{v}_{\mathrm{g}}}{\left|\vec{v}_{\mathrm{g}}\right|}
$$

Tresca donne

$$
\tau=-m \frac{\sigma_{0}}{\sqrt{3}} \frac{\vec{v}_{\mathrm{g}}}{\left|\vec{v}_{\mathrm{g}}\right|}
$$

Si nous appliquons les lois (20) ou (21), nous obtenons une discontinuité de la contrainte de scission au point neutre. Afin d'éviter cette discontinuité, on a souvent recours à une régularisation qui donne :

$$
\tau=-\min \left\{\mu \sigma_{n}, \frac{\sigma_{0}}{\sqrt{3}}\right\} \frac{\left|\vec{v}_{\mathrm{g}}\right|}{\sqrt{v_{\mathrm{g}}^{2}+k \omega^{2}}}
$$

où $k$ est un coefficient de régularisation et $\omega$ est une vitesse de référence égale à la vitesse linéaire des cylindres de travail. Ce modèle est appelé modèle de Coulomb régularisé.

On peut aussi utiliser, notamment à chaud, une loi de frottement dépendant des vitesses avec une relation de type Norton-Hoff :

$$
\tau=-\alpha v_{\mathrm{g}}^{\mathrm{p}}
$$

\subsection{Loi de comportement}

Les tenseurs des contraintes et déformations sont dans ce cadre et en supposant la tôle suffisamment large pour pouvoir écrire $\varepsilon_{2}=0$, de la forme

$$
\sigma_{i j}=\left[\begin{array}{ccc}
\sigma_{1} & 0 & 0 \\
0 & \sigma_{2} & 0 \\
0 & 0 & \sigma_{3}
\end{array}\right] \varepsilon_{i j}=\left[\begin{array}{ccc}
-\varepsilon_{3} & 0 & 0 \\
0 & 0 & 0 \\
0 & 0 & \varepsilon_{3}
\end{array}\right]
$$

Pour une loi de comportement donnée, la condition de déformation plane reportée dans la loi d'évolution plastique permet de calculer la contrainte transversale $\sigma_{2}$ en fonction de $\sigma_{1}$ et $\sigma_{3}$. La contrainte équivalente $\hat{\sigma}$ ou $\bar{\sigma}$ peut alors s'écrire

$$
\hat{\sigma}=C_{0}\left|\sigma_{1}-\sigma_{3}\right|
$$

où $C_{0}$ est une constante dépendant des constantes d'anisotropie.

De même en reportant dans les expressions de la déformation équivalente $\dot{p}$ on obtiendra

$$
\dot{p}=\frac{1}{C_{0}^{\prime}}\left|\dot{\varepsilon}_{3}\right|
$$

La loi de d'écoulement plastique ou viscoplastique s'écrira donc finalement :

$$
\begin{aligned}
& C_{0}\left(\sigma_{1}-\sigma_{3}\right)=Y\left(\frac{1}{C_{0}^{\prime}}\left|\varepsilon_{3}\right|\right) \\
& C_{0}\left(\sigma_{1}-\sigma_{3}\right)=A\left(\frac{1}{C_{0}^{\prime}}\right)^{m+n} \varepsilon_{3}^{n} \dot{\varepsilon}_{3}^{m}
\end{aligned}
$$

Reste à calculer dans chaque cas les deux constantes $C_{0}$ et $C_{0}^{\prime}$.

En plasticité associée $C_{0}=C_{0}^{\prime}$ et on obtient : - dans le cas isotrope complet

$$
C_{0}=\frac{\sqrt{3}}{2}
$$

- dans le cas isotrope transverse

$$
C_{0}=\sqrt{\frac{2+r}{2(1+r)}}
$$

- dans le cas orthotrope

$$
C_{0}=\sqrt{\frac{1+r_{1}+r_{2}}{\left(1+r_{1}\right)\left(1+r_{2}\right)}}
$$

- dans le cas orthotrope non-quadratique

$$
C_{0}=\sqrt[m]{\frac{1+r_{1}+r_{2}}{\left(1+r_{1}\right)\left(1+r_{2}\right)}}
$$

- dans le cas de la plasticité non-associée non-quadratique

$$
C_{0}^{\prime}=\sqrt[m]{\frac{1+\bar{r}_{1}+\bar{r}_{2}}{\left(1+\bar{r}_{1}\right)\left(1+\bar{r}_{2}\right)}}
$$




\subsection{Schéma de calcul}

Le point neutre est défini par $v_{\mathrm{g}}=0, v=\Omega R$. L'hypothèse d'une répartition de vitesses uniforme dans chaque section combinée à la condition d'incompressibilité plastique impose la conservation du débit

$$
v h=\text { const. }=\Omega R h_{n}
$$

Une fois le point neutre connu on peut déterminer les vitesses le long de l'emprise et donc la vitesse de déformation $\dot{\varepsilon}_{3}$. La détermination de ce point neutre joue donc un rôle essentiel dans la résolution du problème.

Il reste alors à intégrer l'équation de Karman en utilisant pour $\left(\sigma_{1}-\sigma_{3}\right)$ les expressions obtenues en $(3-2)$ et pour $\tau$ la loi de frottement en $(3-1)$. Les conditions aux limites sont données par les tensions inter cages et donc par les valeurs des contraintes longitudinales à l'entrée et à la sortie de l'emprise. Le schéma de résolution classique consiste à résoudre cette équation

- à partir de l'entrée pour $x$ croissant

- à partir de la sortie pour $x$ décroissant.

Le point neutre est alors obtenu par l'intersection des deux courbes associées. Cette procédure fonctionne sans problèmes pour le comportement indépendant des vitesses pour le comportement (plasticité) comme pour le frottement (Coulomb ou Tresca). Elle n'est plus applicable par contre lorsque la vitesse - et donc la position du point neutre - intervient explicitement c'est-à-dire en viscoplasticité ou lorsque la loi de frottement dépend des vitesses.

Nous avons donc utilisé une autre technique de résolution déterminant le point neutre par une méthode $\mathrm{du}$ tir. Pour une position donnée du point neutre $x_{n}$, on calcule la contrainte longitudinale $\sigma_{1}$ par intégration de l'équation de Karman en partant de l'entrée et on détermine ainsi sa valeur à la sortie. Il suffit ensuite d'ajuster la valeur de $x_{n}$ pour faire coïncider cette valeur à celle donnée. Techniquement nous avons utilisé la méthode de Runge-Kutta pour intégrer l'équation de Karman et la méthode de la sécante pour ajuster la position du point neutre. Il en résulte un calcul efficace et rapide qui permet de tester aisément différentes hypothèses.

\section{Analyse paramétrique}

Pour dégager l'importance des divers paramètres constitutifs introduits dans nos modèles il est intéressant de mener une analyse paramétrique de leur influence sur le résultat. Nous nous intéressons principalement pour fixer les idées à la pression moyenne, valeur moyenne de $\sigma_{3}$ le long de l'emprise, et représentative en première approximation de la charge verticale.

\subsection{Cadre viscoplastique}

Cet état correspond au laminage des métaux à haute température [8]. Le modèle de Norton Hoff est utilisé dans ce cas. Il dépend donc des coefficients $K$ et $m$. L'influence $(m=0,2)$

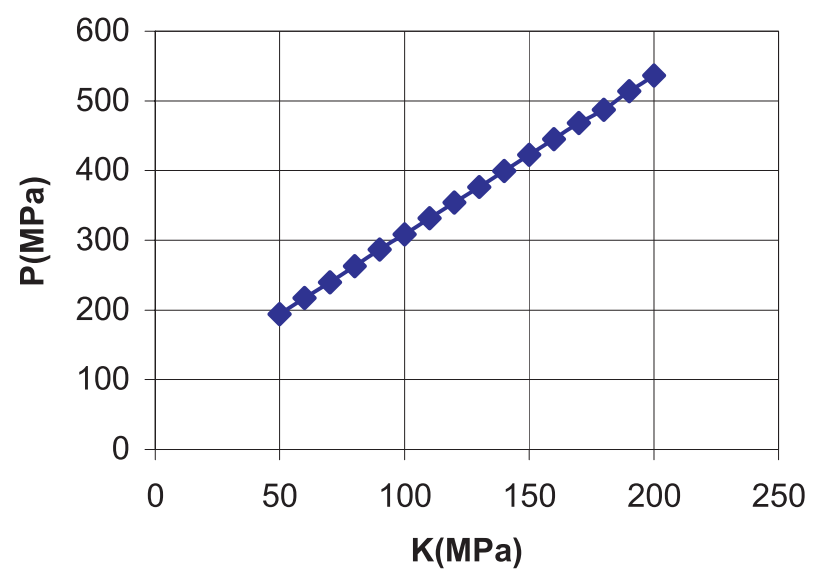

Fig. 1. Évolution des pressions en fonction de $K$.

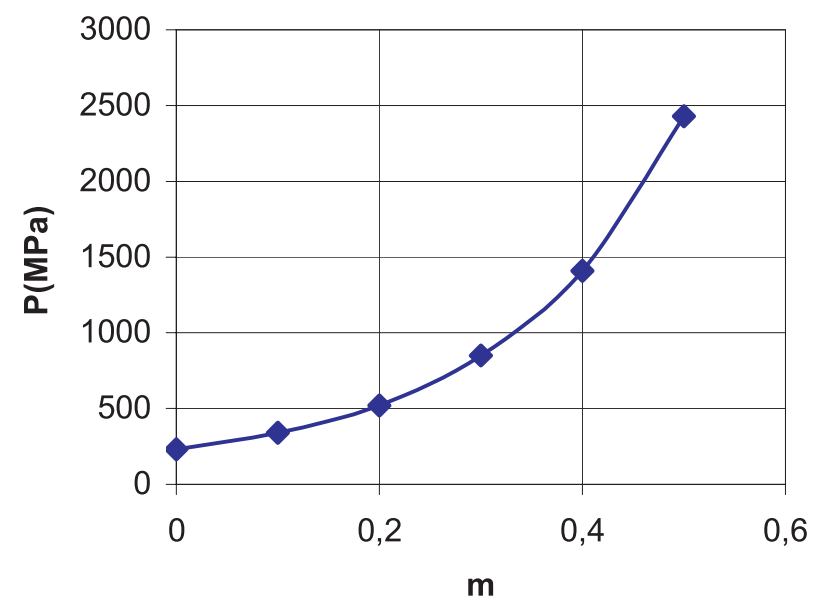

Fig. 2. Évolution des pressions en fonction du coefficient de sensibilité à la vitesse de déformation $(m)$.

de la vitesse est dans ce cas prise en compte. La loi de comportement est donnée par (16) et nous prenons :

$$
\begin{aligned}
R_{\text {cyl }} & =250 \mathrm{~mm} \\
h_{0} & =4 \mathrm{~mm}, \quad h_{\mathrm{s}}=3 \mathrm{~mm} \\
\Omega & =40 \mathrm{rad} . \mathrm{s}^{-1}
\end{aligned}
$$

La loi de frottement utilisée est celle de Norton Hoff (21).

$$
\alpha=5,7 \times 10^{7}, \quad p=0,2 .
$$

Les contraintes longitudinales à l'entrée et à la sortie sont nulles, $\sigma_{1 \mathrm{e}}=\sigma_{1 \mathrm{~s}}=0$.

La viscoplasticité avec écrouissage correspond au laminage à chaud à température moyenne ou à grande vitesse de déformation. Dans ce cas, les valeurs de $m$ (coefficient de sensibilité à la vitesse de déformation) et $n$ (coefficient d'écrouissage) ainsi que la déformation et la vitesse de déformation revêtent une importance primordiale. La loi de comportement est donnée par (17).

Les résultats présentés sur les figures ci-dessus montrent que l'identification de la loi de comportement 


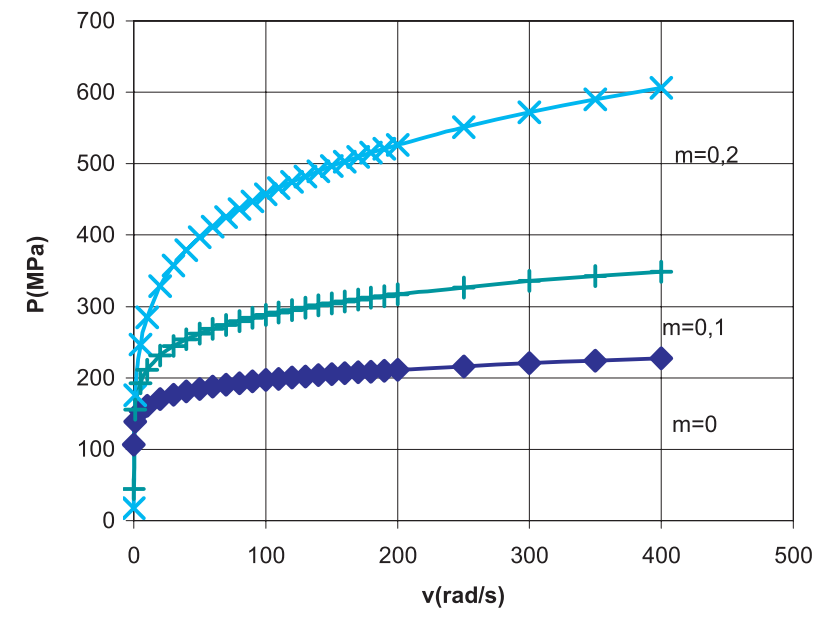

Fig. 3. Évolution des pressions en fonction de la vitesse des cylindres de travail.

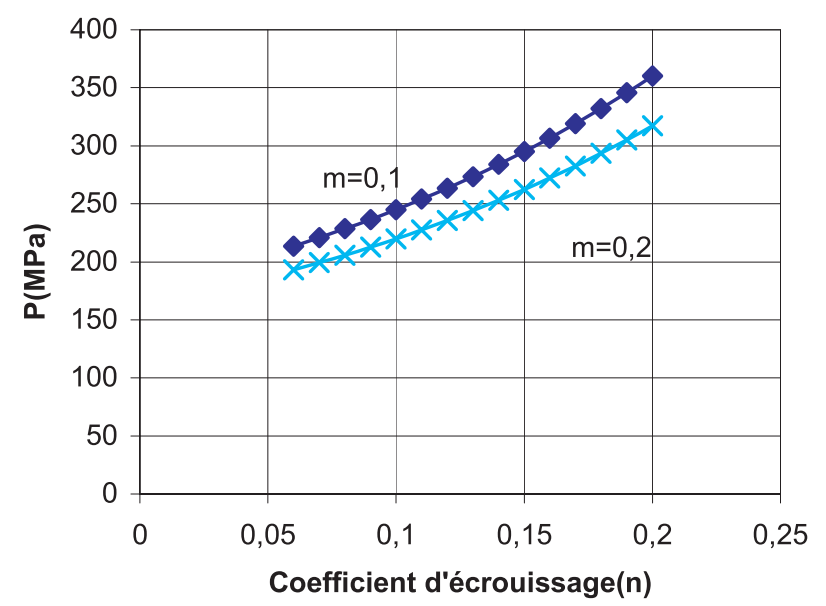

Fig. 4. Évolution des pressions en fonction du coefficient d'écrouissage pour différents coefficients de sensibilité à la vitesse de déformation $(m=0,1$ et $m=0,2)$.

viscoplastique doit être d'autant plus précise que les vitesses sont plus grandes.

\subsection{Cadre plastique}

Ce cadre correspond au laminage à froid.

$$
\begin{aligned}
R_{\text {cyl }} & =250 \mathrm{~mm} \\
h_{0} & =1 \mathrm{~mm}, \quad h_{\mathrm{f}}=0,77 \mathrm{~mm} \\
\Omega & =40 \mathrm{rad} . \mathrm{s}^{-1}
\end{aligned}
$$

avec une loi de Swift

$$
Y(p)=643\left(\varepsilon_{0}+p\right)^{n} \quad \varepsilon_{0}=0,01 \quad n=0,19
$$

$\sigma_{1 \mathrm{e}}$ et $\sigma_{1 \mathrm{~s}}$ sont les contraintes longitudinales à l'entrée et à la sortie de l'emprise.

$$
\sigma_{1 \mathrm{e}}=\sigma_{1 \mathrm{~s}}=0,1 Y(p)
$$

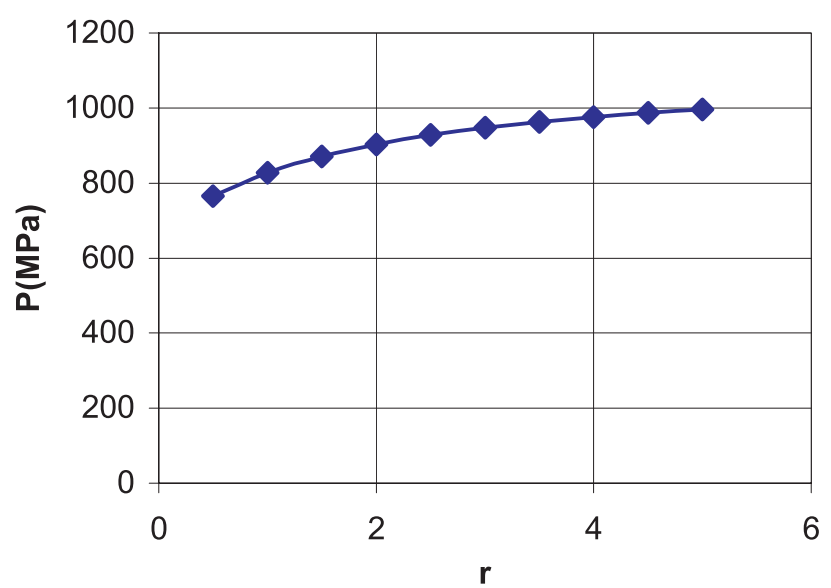

Fig. 5. Évolution des pressions en fonction du coefficient de Lankford.

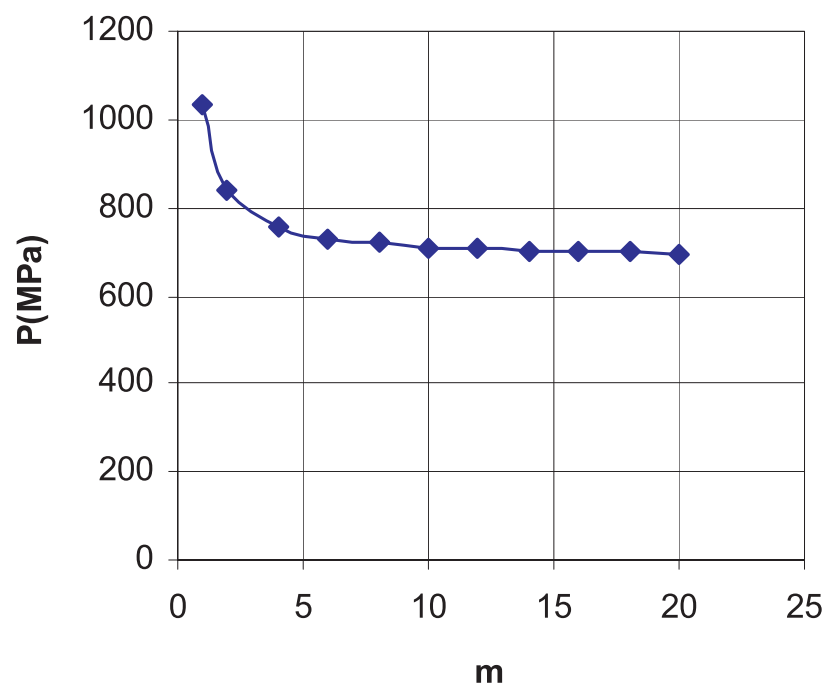

Fig. 6. Évolution des pressions en fonction du coefficient de forme $m$.

Les résultats présentés dans les figures 5-7 montrent combien les résultats du calcul au laminage sont sensibles à l'anisotropie (à travers le coefficient de Lankford) et à la nature du critère (valeur de $m$ ).

Ces résultats étaient difficilement prévisibles dans la mesure où les coefficients de Lankford ont été définis en liaison avec l'emboutissage (donc avec une $3^{\mathrm{e}}$ direction, l'épaisseur, libre de contrainte alors qu'ici la $3^{\mathrm{e}}$ direction est au contraire à déformation imposée nulle) Ceci peut en partie expliquer qu'un coefficient de Lankford élevé rende le laminage plus difficile.

L'augmentation du coefficient de forme $m$ donne des pressions moins importantes. Cela est très significatif pour $1<m<5$. On voit de ce fait que les résultats sont très sensibles à l'anisotropie et au coefficient de forme $(m)$. 


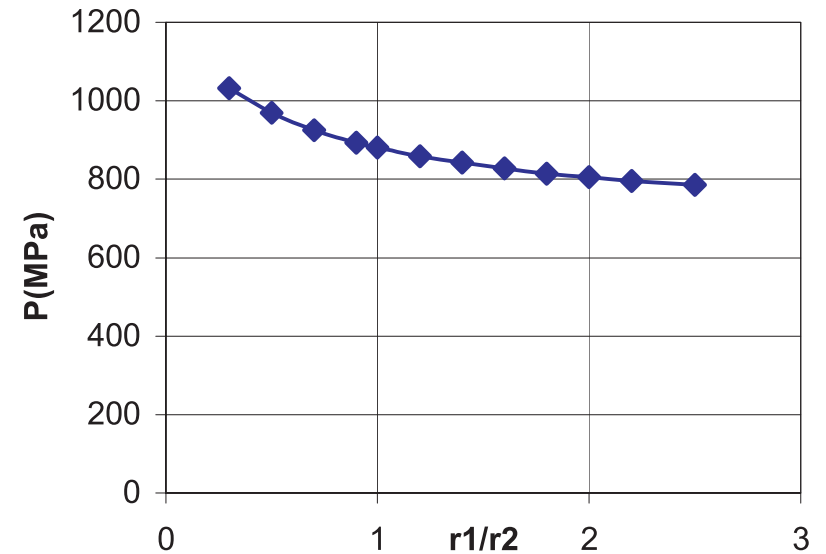

Fig. 7. Évolution des pressions en fonction du rapport $\frac{r_{1}}{r_{2}}$.

\section{Application}

\subsection{Laminage à froid}

Les résultats précédents montrent l'influence des différents paramètres mais sont difficilement comparables du fait qu'ils se référent à des matériaux différents. Pour comparer les modèles entre eux il faut au contraire partir d'un matériau donné et utiliser différents modèles susceptibles d'être utilisés pour le représenter.

Nous disposons pour cela des résultats de A. Khalfallah $[4,9]$ pour l'identification des lois de comportement plastique concernant trois tôles.

Sur les bases de données expérimentales où trois types d'essais ont été réalisés (traction simple, traction plane et expansion biaxiée), A. Khalfallah a identifié les lois de comportement de ces trois tôles dans les cadres de la plasticité associée et non-associée avec critère quadratique dans un premier temps et d'une loi de normalité associée avec critère non-quadratique dans un second.

Deux stratégies d'identification ont été utilisées. La première, classique, considère les essais expérimentaux comme homogènes et la seconde combine un code de calcul et une procédure d'optimisation pour prendre en compte la non-homogénéité des essais

Pour les trois tôles, la loi d'écrouissage utilisée est celle de Swift.

Tôle 1 : D280 acier micro allié à haute limite d'élasticité. $K=643 \times 10^{6}, \varepsilon_{0}=0,007, n=0,19$.

Tôle 2 : IF acier sans interstitiels.

$$
K=580 \times 10^{6}, \quad \varepsilon_{0}=0,004, \quad n=0,26 .
$$

Tôle 3 : ES acier extra doux, calmé à l'aluminium. $K=$ $557 \times 10^{6}, \varepsilon_{0}=0,004, n=0,23$.

Il est à remarquer que la tôle 2 est la plus fortement anisotrope $\left(r_{\text {hom }}=1,25, r_{\text {inv }}=1,71\right)$ suivie des deux autres tôles où pour la première tôle $1 r_{\text {hom }}=0,93$ et $r_{\mathrm{inv}}=1,21$ et la troisième $r_{\mathrm{hom}}=1,05$ et $r_{\mathrm{inv}}=1,19$. Ces résultats ont été obtenus dans le cadre isotrope transverse où l'axe de symétrie coïncide avec la direction normale à la tôle, en considérant les essais comme homogènes et non-homogènes.

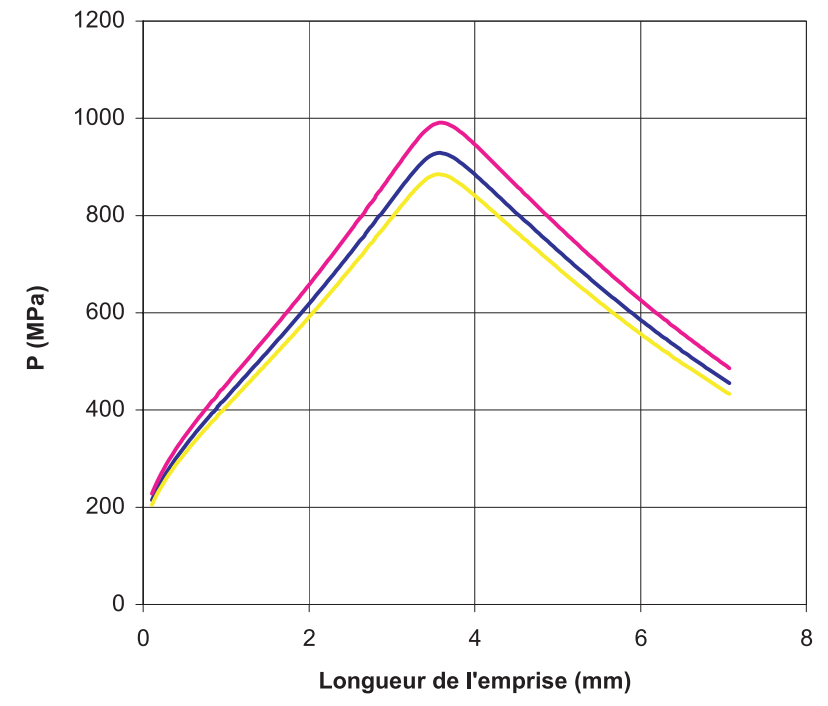

Fig. 8. Évolution des pressions le long de l'emprise dans les cas isotrope, isotrope transverse et orthotrope, $p_{\text {iso }}<p_{\text {iso transv }}<$ $p_{\text {orthotrope }}$

Tôle 2

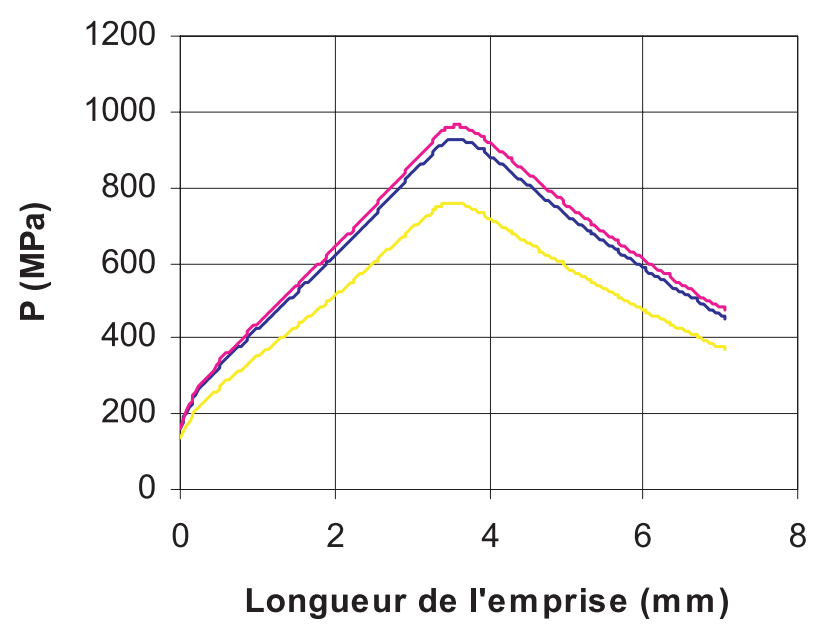

Fig. 9. Évolution des pressions le long de l'emprise dans les cas quadratiques et non-quadratique $(m=8), p_{m=8}<$ $p_{\text {hom }, m=2}<p_{\text {inv }, m=2}$.

La figure 8 montre bien l'importance de la prise en compte de l'anisotropie. En effet, nous voyons bien la différence de résultats obtenus dans ces différents cadres.

Dans la figure 9 nous confirmons ce qui a été dit plus haut, à savoir que si le coefficient de forme $m$ augmente, les pressions diminuent sensiblement, d'où la nécessité de l'identifier avec le plus grand soin.

Ces résultats, sont difficiles à interpréter. Un élément d'interprétation se trouve dans la nature de la sollicitation de cisaillement simple, sollicitation sévère et qui en particulier exacerbe l'importance du coefficient $m$ (c'est en fait dans le cas isotrope que se situent les plus grandes différences entre Von Mises $(m=2)$ et Tresca $(m=\infty)$.

Nous comparons les résultats obtenus lors du calcul des pressions pour les trois tôles dans le cadre orthotrope. 
Tôle 3

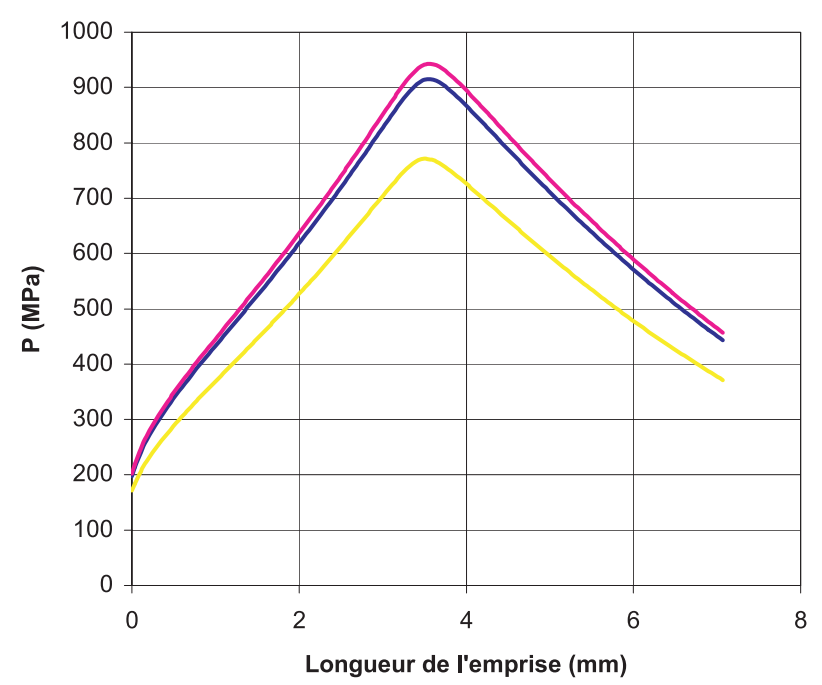

Fig. 10. Évolution des pressions le long de l'emprise dans les cas quadratiques et non-quadratique $(m=8), p_{m=8}<$ $p_{\text {inv }, \mathrm{m}=2}<p_{\text {hom }, m=2}$.

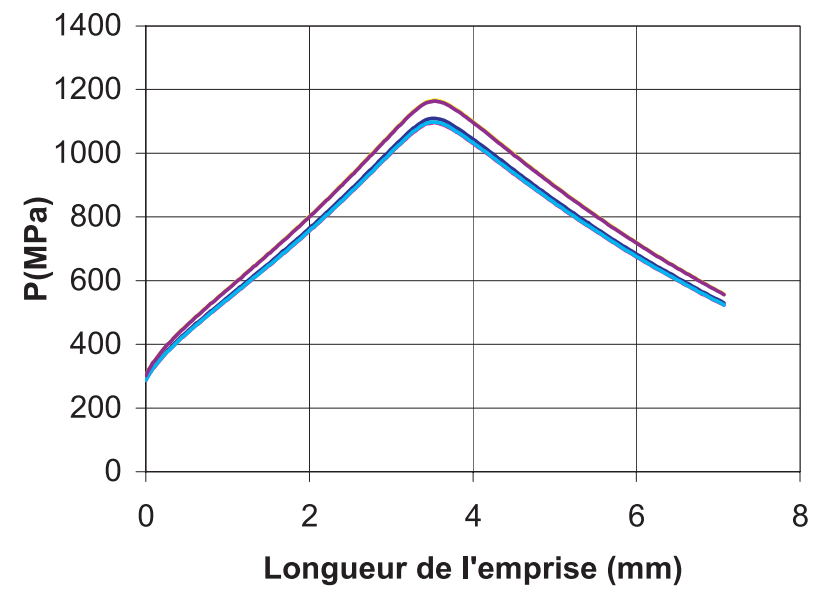

Fig. 11. Évolution des pressions le long de l'emprise dans différents cadres de comportement, tôle $1, p_{\text {iso }}<$ $p_{\text {hom }_{\text {non associée }}}<p_{\text {hom }}<p_{\text {inv }_{\text {non associée }}}<p_{\text {inv }}$.

Il s'avère que dans tous les cas, la démarche d'identification inverse donne des résultats nettement différents avec la méthode homogène. Par contre, la différence entre les résultats obtenus en plasticité associée et non-associée est d'autant plus significative que la tôle est anisotrope. Cela conforte l'idée qu'il faut se méfier de la plasticité associée pour les tôles fortement laminées à froid du fait de leurs anisotropies. Ainsi, pour la tôle une qui est très faiblement anisotrope, la plasticité associée et non-associée donnent pratiquement les mêmes résultats, contrairement aux autres tôles.

\subsection{Laminage à chaud}

Sur la base d'essais de compression à chaud où les conditions de lubrification à l'interface échantillon-bas de

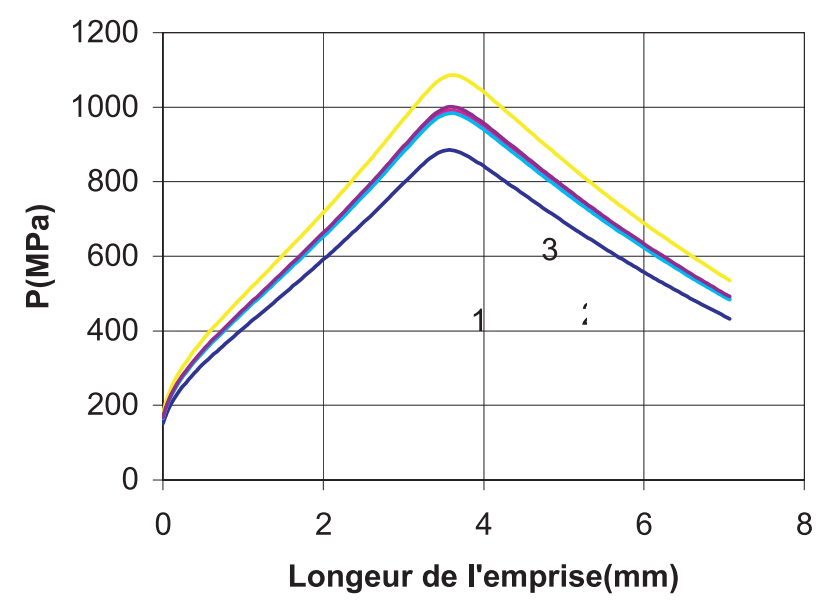

Fig. 12. Évolution des pressions le long de l'emprise dans différents cadres de comportement, tôle $2, p_{\text {isotrope }}<$ $p_{\text {hom }_{\text {non associée }}}<p_{\text {hom associée }}<p_{\text {inv non }_{\text {associée }}}<p_{\text {inv }}$.

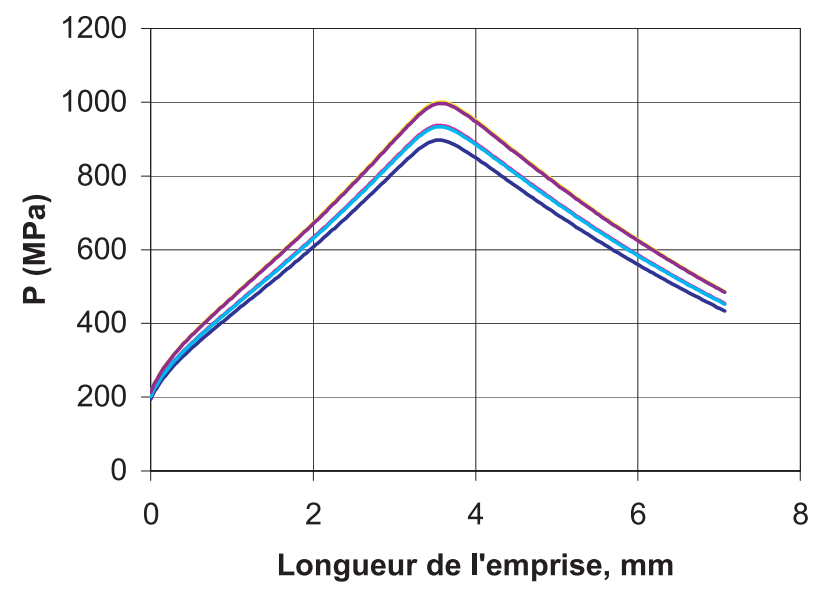

Fig. 13. Évolution des pressions le long de l'emprise dans différents cadres de comportement, tôle $3, p_{\text {iso }}<$ $p_{\text {hom }_{\text {non associée }}}<p_{\text {hom }}<p_{\text {inv }_{\text {non associée }}}<p_{\text {inv }}$.

la presse permettent d'obtenir des essais homogènes où la déformation peut atteindre l'unité, A. Darsouni [10] a identifié les paramètres rhéologiques que sont le coefficient de sensibilité à la vitesse de déformation $m$ et le coefficient d'écrouissage $n$ à différentes températures; et cela pour des nuances d'acier industrielles en brut de coulée (B .C) et brut de laminage (B .L).

$$
\begin{aligned}
& n=\left(\frac{\partial \ln \sigma}{\partial \ln \varepsilon}\right)_{T, \varepsilon} \\
& m=\left(\frac{\partial \ln \sigma}{\partial \ln \dot{\varepsilon}}\right)_{T, \dot{\varepsilon}}
\end{aligned}
$$

Il a aussi identifié les lois de comportement avec deux démarches différentes, la première suppose la vitesse constante et la seconde la contrainte constante. Nous présentons ces résultats dans le tableau suivant.

Dans la figure qui suit, nous présentons l'évolution des contraintes longitudinales le long de l'emprise en utilisant différentes valeurs du coefficient de sensibilité 


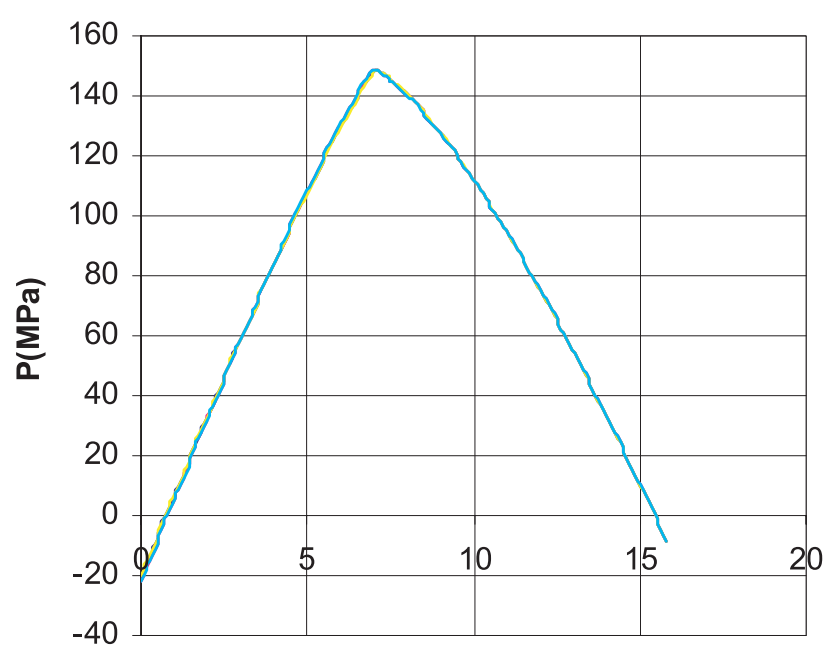

Longueur de l'emprise $(\mathrm{mm})$

Fig. 14. Évolution des pressions le long de l'emprise.

\begin{tabular}{ccccc}
\hline$T$ & $m$ & $m$ & $m$ loi & $m$ exp \\
$(\mathrm{C})$ & $\begin{array}{c}m \\
\text { vitesse } \\
\text { const }\end{array}$ & $\begin{array}{c}\text { contrainte } \\
\text { const }\end{array}$ & $\begin{array}{c}\text { puissance } \\
\text { pons }\end{array}$ & \\
\hline 700 & 0,124 & 0,154 & 0,154 & 0,154 \\
750 & 0,156 & 0,167 & 0,150 & 0,169 \\
800 & 0,144 & 0,159 & 0,140 & 0,117 \\
850 & 0,136 & 0,137 & 0,137 & 0,137 \\
900 & 0,167 & 0,167 & 0,167 & 0,167 \\
950 & 0,161 & 0,161 & 0,161 & 0,161 \\
1000 & 0,165 & 0,165 & 0,165 & 0,165 \\
\hline
\end{tabular}

à la vitesse de déformations identifiées par ajustement et expérimentalement; et cela, en considérant la vitesse constante puis la contrainte constante. Le problème rencontré pour exploiter convenablement ces résultats se situe dans la différence importante des vitesses entre le laminage et les essais de compression utilisés. Cela est dû au fait que ce coefficient est fonction des vitesses utilisées. Il s'avère que les résultats sont pratiquement insensibles à ces différentes démarches de détermination du coefficient de sensibilité à la vitesse de déformation.

\section{Conclusions}

Le caractère ouvert et évolutif de nos programmes informatiques ainsi que la vitesse d'exécution du programme principal nous a permis de tester efficacement et agréablement différents modèles de comportement plastiques et viscoplastiques en laminage. Dans le cadre plastique (laminage à froid), nous confirmons la nécessité de prendre en compte l'anisotropie et nous avons montré la forte influence du coefficient de forme $m$ qu'il est toutefois encore bien difficile d'identifier avec précision. Pour le cadre viscoplastique l'identification du coefficient de sensibilité à la vitesse de déformation doit être d'autant plus précise que les vitesses augmentent.
L'implantation des résultats d'identification de A. Khalfalla et de A. Darsouni nous a permis de tirer quelques conclusions très utiles pour une meilleure maîtrise du laminage. Nous confirmons en particulier la nécessité de passer à la plasticité non-associée lorsque les tôles sont fortement anisotropes ainsi que la forte sensibilité au coefficient de forme. Il s'avère aussi que les deux stratégies d'identification (démarche homogène et nonhomogène) donnent des résultats nettement différents au laminage. Il convient toutefois d'insister sur le fait que ces résultats d'identification ont été obtenus dans une optique emboutissage, et ne sont pas nécessairement pertinents en vue du laminage. Il faut également souligner le fait que le laminage et les évolutions de texture qui en résultent sont la cause première de l'anisotropie des tôles. Il n'est donc pas raisonnable de la supposer constante et il faudra au moins prendre en compte son évolution au cours des différentes passes. Des essais et des méthodes d'identification mieux adaptés au laminage devront être développés pour poursuivre ce travail.

Par contre, la sensibilité aux différentes démarches de détermination du coefficient de sensibilité à la vitesse de déformation semble négligeable dans le cadre viscoplastique (laminage à chaud).

\section{Références}

[1] A. Dogui, F. Sidoroff, Large strain formulation of anisotropic elasto-plasticity for metal forming, Comp. Meth. for Predicting Material processing defects, ed. M. Predeleanu, Elsevier, 1987, pp. 81-92

[2] F. Sidoroff, Modèles phénoménologiques, Physique et mécanique de la mise en forme, École d'été d'Oléron, 1990, pp. 297-307

[3] P. Montmitonnet, Laminage à froid, Modélisation, M616, Technique de l'ingénieur, 1990

[4] A. Khalfallah, Identification des lois de comportement élastoplastiques par essais inhomogènes et simulations numériques, thèse de doctorat soutenue à la faculté des sciences de Tunis, février 2004

[5] A. Znaidi, Identification des lois de comportement élastoplastiques, thèse de doctorat soutenue à la faculté des sciences de Tunis, février 2004

[6] P. Montmitonnet, Lois de frottement et déformation plastique, Matériaux et Techniques, 1993, pp. 8-29

[7] J. Oudin, Approches expérimentales et numériques des conditions de contact et de frottement, Physique et mécanique de la mise en forme, École d'été d'Oléron, 1990, pp. 406-429

[8] C. David, Modélisation numérique par éléments finis du forgeage à chaud, Physique et mécanique de la mise en forme, École d'été d'Oléron, 1990, pp. 542-548,

[9] A. Khalfallah, Anisotropic parametre identification using inhomogeneous tensile test, European J. Mechanics A/Solids 21 (2002) 927-942

[10] A. Darsouni, Étude du comportement thermomécanique d'un acier micro allié à des températures intermédiaires (700-1200 C), thèse de doctorat d'état présentée à l'université Badii-Mokhtar (Annaba), juin 2003 